\title{
Photometric study of the short-period RS Canum Venaticorum binary RT Andromedae ${ }^{\star}$
}

\author{
Li-yun Zhang ${ }^{1,2}$ and Sheng-hong $\mathrm{Gu}^{1}$ \\ 1 National Astronomical Observatories/Yunnan Observatory, Joint laboratory for optical astronomy, Chinese Academy of Sciences, \\ Kunming, PR China \\ e-mail: zhangliyun2000.student@sina.com \\ 2 Graduate School of Chinese Academy of Sciences, Beijing 100039, PR China \\ e-mail: zhangliyun2000.student@sina.com
}

Received 18 November 2006 / Accepted 11 April 2007

ABSTRACT

\begin{abstract}
Context. Long-term photometric observations of cool stars can reveal the evolution and activity cycles of the stellar active region. Furthermore, studying the stellar activity provides opportunities for understanding of stellar dynamo and valuable constraints for stellar dynamo theory.

Aims. We analyze our observational data and discuss light-curve variability due to starspots on both a short and long time scale, especially the short-term variation. At the same time, we accumulate some results from the literature to discuss active-region evolution and activity cycles.

Methods. By analyzing the light curves using the Wilson-Devinney program, the photometric solution of the system is obtained and the starspot parameters are also derived. Using the phase-dispersion minimization method, we infer the activity cycle of RT And. Results. The case of two spots being on the primary is most successful in reproducing the light curve distortion of RT And in 2004. Although the light curves in 1999 and 2005 do not have sufficient phase coverage, we still could use the spot model to explain their light-curve variation successfully based on the photometric solution in 2004. Comparing the light curves of 1999, 2004, and 2005, the light curve distortion changes on both short and long time scales, especially the significant variation around the secondary minimum on a time scale of two months. Analysis of the longitude of spots within the belt around $270^{\circ}$ suggests that the activity cycle of RT And may be $6.69 \pm 0.80$ years.
\end{abstract}

Key words. stars: active - binaries: eclipsing - stars: individual: RT And - starspots

\section{Introduction}

The active star RT And (BD+52 $3383 a$, F 8-G 0 V+K 1-3 V) is a short-period eclipsing binary. It has been extensively observed since the beginning of the 20th century due to the variability of the light curve (LC). The first photoelectric LC of RT And was obtained in 1947-1950 by Gordon $(1948,1955)$. She found it appeared variable both nightly and yearly in this shape and showed marked asymmetry in the secondary minimum. Dean (1974) also found these variations in 1962 and 1964. Mancuso et al. (1979a) confirmed that RT And had a highly variable LC from night to night and from year to year. Milano et al. (1981) analyzed all available photoelectric LCs of RT And obtained up to 1978 and proposed that the photometric distortion waves were caused by the spots. Zeilik et al. (1989) successfully explained the photometric distortion waves in the LCs by using dark circular starspots at middle-high latitudes on the primary with the temperature roughly $1100-1200 \mathrm{~K}$ below that of the photosphere. Several authors have explained the distortion waves by the circular starspot model: Hechert (1995, 1996, 1998), Pribulla et al. (2000), and Kjurkchieva et al. (2001).

In earlier photometric solutions of RT And, there were dissensions, especially for the orbital inclination. Two different values of orbital inclination have been derived. One is close to $82^{\circ}$

$\star$ Table 1 is only available in electronic form at http://www. aanda.org
(Payne-Gaposchkin 1946; Mancuso et al. 1979b; Wang \& Lu 1993; Kjurkchieva et al. 2001) and the other is close to $88^{\circ}$ (Dean 1974; Dumitrescu 1974; Milano et al. 1981; Budding \& Zeilik 1987; Zeilik et al. 1989; Heckert 1998). Both values were derived by Arévalo et al. (1995) through analysis of the first infrared $J$ and $K$ LCs of RT And. They prefer the second value on the basis of the morphology of the secondary eclipse. Furthermore, UBVRIJK LCs without maculation effects and radial velocity curves of both components were analyzed by Pribula et al. (2000) using the Wilson-Devinney program. They also derived the two values for orbital inclination and preferred the high orbital inclination due to the total eclipse of the secondary minimum, which was likewise observed as a test.

Until now, there have been several spectroscopic study for RT And. The first radial velocity curves of both components of RT And were derived by Payne-Gaposchkin (1946). Highquality spectroscopy of RT And was obtained by Popper (1994) in 1989-1993. He derived the radial velocities by the advanced cross-correlation procedure that counts theoretical corrections for rotational velocities, line strength ratios, line separations, proximity effects of radiation, and tidal distortion and determined $K_{1}=135.4 \pm 0.6 \mathrm{~km} \mathrm{~s}^{-1}$ and $K_{2}=185.1 \pm 1.0 \mathrm{~km} \mathrm{~s}^{-1}$. Further spectroscopy of RT And was obtained by Kjurkchieva et al. (2001). They determined $K_{1}=130 \mathrm{~km} \mathrm{~s}^{-1}$ and $K_{2}=$ $175.8 \mathrm{~km} \mathrm{~s}^{-1}$ by measuring the double profiles of the lines $\mathrm{H}_{\alpha}$ and FeI $6678 \AA$ A. 


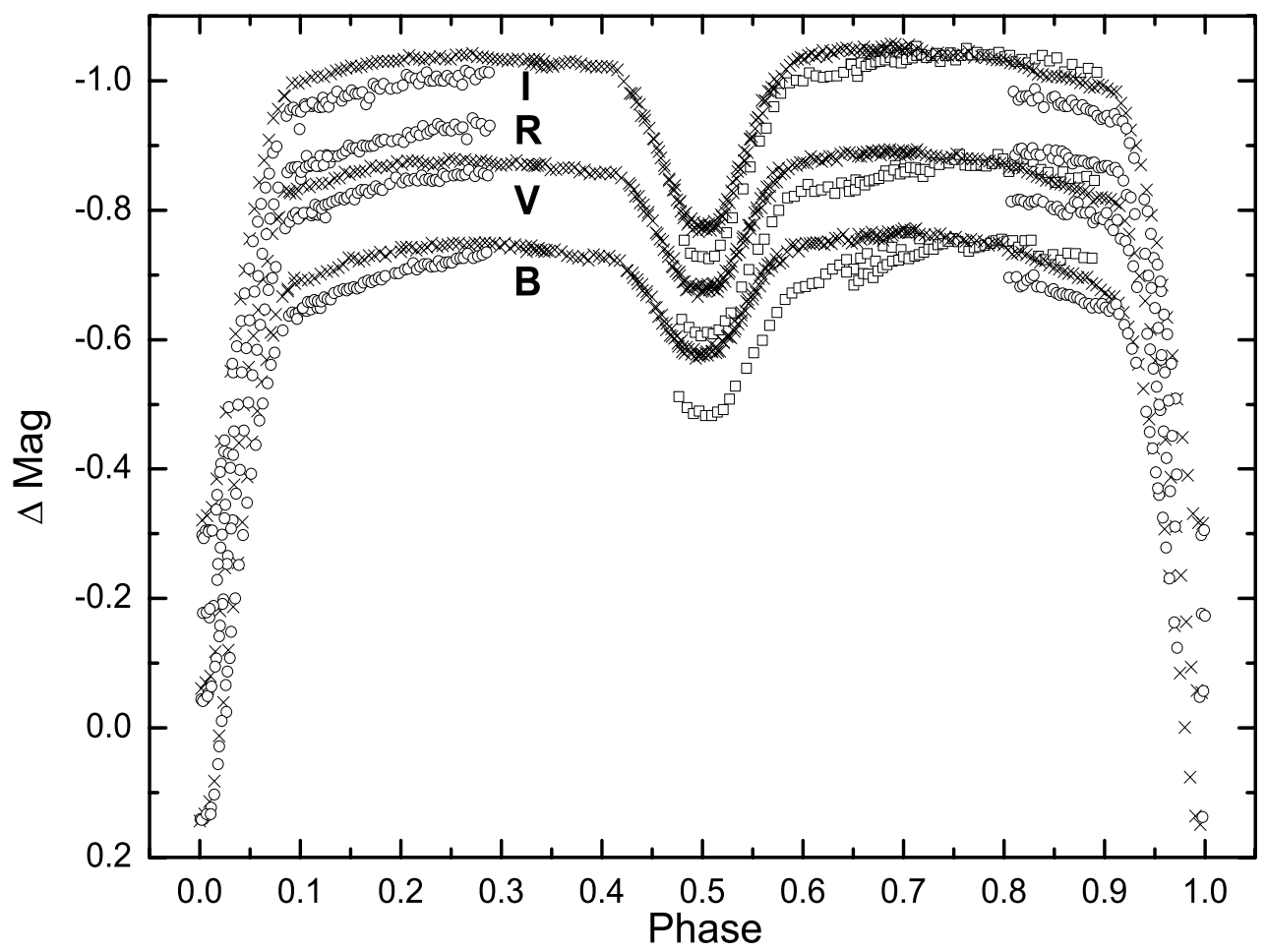

Fig. 1. $B, V, R$, and $I$ observations of RT And at Yunnan Observatory. Circles (o) indicate 10.13-15.1999, crosses $(\times)-11.03-07.2004$, and squares (口) $-1.02-04.2005$.

In this paper, we present new $B, V, R$, and $I$ CCD LCs of RT And. We have analyzed our observational data using the version 2003 of Wilson-Devinney code and discuss LC's variability due to starspots on short and long time scales, especially the short time-scale variation. At the same time, we accumulate some results from the literature to discuss the activeregion evolution and activity cycle.

\section{Observation}

The new photometric observations were made on two nights (Oct. 13 and 15, 1999), four nights (Nov. 3, 4, 5, and 7, 2004), and two nights (Jan. 2 and 4, 2005) with the $1 \mathrm{~m}$ Cassegrain telescope of Yunnan Observatory, China. The photometer was equipped with a $1024 \times 1024$ pixel CCD and the $B V R I$ filters were used. The observation in 1999 was made in $B, V, R$, and $I$ passbands, while those in 2004 and 2005 were made only in $B$, $V$, and $I$. All observed CCD images were reduced by means of the IRAF Package in the standard fashion. BD $+52^{\circ} 3385$ and $\mathrm{BD}+52^{\circ} 3384$ served as comparison and check stars, respectively. The magnitude values of RT And, the comparison, and the check star were determined using the Apphot sub-package of IRAF. Figure 1 shows all the LCs of RT And. The errors of individual points do not exceed $0.008 \mathrm{mag}$ in $B, V, R$, and $I$.

For our observations, the times of the minima of our LCs are determined by means of parabola fitting. All published photoelectric and CCD minimum times in recent decades were collected from the literature and analyzed using the linear leastsquare method. The four secondary minimum times (deviating too much) whose $\mathrm{O}-\mathrm{C}$ values are higher than 0.0025 were omitted because they might be influenced by spots and shifted. They are listed under the line at the end of the compilation of the minima times. The remaining minimum times listed in Table 1 were fitted again using the linear least-square method, and a new linear ephemeris formula was obtained as follows:

Min.I $=\mathrm{JD}(\mathrm{Hel}) .2451498.4677( \pm 0.0001)$ +0 d $62892846( \pm 0.00000009) E$.

We defined 2451498.4680 as the epoch zero point. The $\mathrm{O}-\mathrm{C}$ values listed in Table 1 were calculated with our new ephemeris.

For our new observations, the phases of data points are calculated using the above formula (1). For the sake of comarison, our observations are displayed in Fig. 1 where $\Delta$ Mag represents the differential magnitude between RT And and the comparison. Different kinds of symbols represent the different observations. No vertical shift has been applied to our observational data in Fig. 1. It is evident that the LCs of RT And have changed among 1999, 2004, and 2005, as seen in the next section.

\section{Light curve analysis}

Because our data obtained in 2004 have high time resolution and full phase coverage, photometric solution of RT And might be obtained for this dataset using the 2003 version of the Wilson-Devinney program (Wilson \& Devinney 1971; Wilson \& Van Hamme 2004), as seen in the Sect. 3.1. Although the LCs in 1999 and 2005 do not have sufficient phase coverage, we still try to use the spot model to explain LC variation based on the photometric solution in 2004. Analysis of the LCs in 1999 and 2005 is given in the Sect. 3.2. In order to discuss the spot evolution, in the Sect. 3.3 we analyze the LC variation.

\subsection{Analysis of the light curve in 2004}

From the asymmetric LCs outside the eclipse in 2004, we find there are two depressions in the phase ranges $0.2-0.4$ (spot1) 
and 0.8-0.95 (spot2). Because of having no simultaneous spectroscopic observation, we are not able to tell whether the spots are located on the primary or secondary components. To consider all possibilities, we have performed four solutions (case1: spot 1 and spot 2 on the primary, case 2 : spot 1 on the secondary and spot 2 on the primary, case 3 : spot 1 on the primary and spot 2 on the secondary, case 4 : spot 1 and spot 2 on the secondary).

Individual points in $B, V$, and $I$ bands are used directly for photometric analysis, and the three LCs are simultaneously solved. Mode 2 of Wilson-Devinney code (appropriate for detached binaries) is employed assuming synchronous rotation and zero eccentricity. Simple treatment is used to compute the reflect effect, and the linear limb-darkening law is used to compute the limb-darkening effect. The bolometric albedo $A_{1}=A_{2}=0.5$, the limb-darkening coefficients $x_{1 B}=0.765 x_{2 B}=0.865$, $x_{1 V}=0.655 x_{2 V}=0.771, x_{1 I}=0.554 x_{2 I}=0.566$, and the gravity-darkening coefficients $g_{1}=g_{2}=0.32$ are set for the primary and the secondary, as usual.

According to the spectral type - effective temperature relation of Johnson (1966), the effective temperature of the spectral type F8V is $T_{1}=6100 \mathrm{~K}$ and of G0V $T_{1}=5900 \mathrm{~K}$. On the other hand, Lang(1992) gives the temperatures of $6200 \mathrm{~K}$ and $6030 \mathrm{~K}$ for these two spectral types, respectively. It is reasonable that we adopt the average of the two temperatures of the primary $6100 \mathrm{~K}$ for F8V and $5965 \mathrm{~K}$ for G0V. Actually we only use the temperature of the primary component $T_{1}=6100 \mathrm{~K}$, because the derived geometric elements did not differ significantly for solutions with the temperature of the primary component at two boundary values $6200 \mathrm{~K}$ and $5900 \mathrm{~K}$ (see Pribulla et al. 2000).

For our observed secondary minima of RT And, it favors the total eclipse corresponding to high inclination. Therefore we choose $i=87.57^{\circ}$ derived by Pribula et al. (2000) and make it a fixed parameter.

From spectroscopic observations of RT And, the mass ratios $q_{1}=0.731$ and $q_{2}=0.739$ have been obtained by Popper et al. (1994) and by Kjurkchieva et al. (2001), respectively. We use the mean of the two mass ratios $q=0.735$ as the value of $q$ and fix it. This mass ratio is similar to the value 0.7374 obtained by analyzing UBVRIJK LCs without maculation effects, together with radial velocity curves of both components by means of WilsonDevinney program (Pribulla et al. 2000).

When performing the differential correction calculation, the adjustable parameters are the temperature of the secondary $T_{2}$, the dimensionless potentials of the two components $\Omega_{1}$ and $\Omega_{2}$, the monochromatic luminosity of the primary $L_{1}$ deriving from the approximate Kurucz atmosphere model option of the Wilson-Devinney program (Kurucz

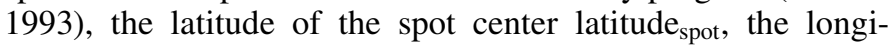

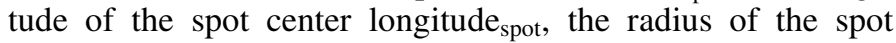
radius $_{\text {spot }}$, and the temperature factor $\left(T_{\text {spot }} / T_{\text {star }}\right)$ of the spot temperaturef $_{\text {spot. }}$ To avoid the correlations among the adjusted parameters, they are divided into three subsets $\left\{T_{2}, \Omega_{1}, \Omega_{2}, L_{1}\right\}$, $\left\{\right.$ latitude $_{\text {spot } 1}$, longitude $_{\text {spot } 1}$, radius $_{\text {spot } 1}$, temperaturef spot $\left.1_{1}\right\}$, and $\left\{\right.$ latitude $_{\text {spot } 2}$, longitude $_{\text {spot } 2}$, radius $_{\text {spot } 2}$, temperaturef $\left._{\text {spot } 2}\right\}$. Then each subset is adjusted separately until three subsets converge.

The procedure of photometric solution was three consecutive steps

1. The first step of our procedure is to adjust the orbital parameters selected in order to get a theoretical LC without maculation effects using those parts of the observed LC that are least affected by the spots - the phase range $0.5-0.7$. We fix in advance the orbital inclination $i=87.57^{\circ}$ and the mass ratio $q=0.735$. We assume the preliminary value for the temperature of the second component $T_{2}=4900 \mathrm{~K}$ corresponding to the spectral type. The preliminary values of $\Omega_{1}$ and $\Omega_{2}$ are taken from photometric solution derived by Pribulla et al. (2000). The luminosity $L_{1}$ of RT And is approximately $4 \pi$ times $l_{1}$, which is the luminosity value per $1 / 4 \pi$ of the area of a sphere centered on the primary at the secondary minimum when the secondary is eclipsed.

At the beginning, we only adjust $L_{1}$ until the output corrections are smaller than the errors of the elements. Then we release the temperature of secondary $T_{2}$ as a free parameter and continue the differential correction calculation until they converge. When calculating the synthetic LCs by means of the Wilson-Devinney LC program, we find the LCs may be accepted basically at the phase $0.5-0.7$, so we do not release $\Omega_{1}$ and $\Omega_{2}$.

2. The second step of our procedure is to adjust the spot parameters in order to fit the rest parts of the LCs, especially, to reproduce the shapes of the distortion outside the eclipses. The preliminary longitudes of the spots are determined from the centers of the depression of LCs. In the Wilson-Devinney notation, the longitude of starspot center is measured counterclockwise (as viewed from above the $+Z$ axis) from the line of star centers, from the 0 to $2 \pi$ radians. In the present paper, it has been transformed to the binary orbital motion notation. The latitude of starspot center is measured from 0 radians at the "north" $(+Z)$ pole to $\pi$ radians at the "south" pole. Zeilik et al. (1989) found that the active region on the primary star tended to occur at middle-high latitude and the temperature was roughly $1100-1200 \mathrm{~K}$ below the photosphere by analyzing the LCs from 1920-1989, so we assume the temperature factor is 0.8 and the latitude of the spot is $45^{\circ}$. We estimate the radius of the spot by making the theoretical LCs to fit the observed ones, especially in the phase ranges of the photometric distortion.

We adjust the two spots separately. At first, we improve the longitude of the spot. Then, because our observations are in three colors, we also try to determine the latitude, the radius, and the temperature factors of the spot for the best fit, even if the area of the spot is highly correlated with its temperature and the latitude of spot is highly correlated with its radius. We adjust the remaining three parameters of the spot until they converge or until the weighted sum of squares of the residual is as low as possible. Finally, we adjust all the spot parameters.

3. The last step is to separately adjust the orbital parameters and spot parameters again until they converge or the theoretical curves fit the observed ones well.

After a lot of runs, four photometric solutions of RT And are derived. They are listed in Table 2, and the theoretical LCs for the spotted solutions are shown in Fig. 2. From these results we find all four solutions fit the distortion of the LCs well. For case1, every spot parameter subset and the orbital parameter subset converge. For case2, the spot 1 parameter subset, the spot 2 parameter subset, and the orbital parameter subset converge. When spot 1 is on the secondary, it is huge in size to produce the required light effect, because the contribution of the secondary component of RT And to the total light is about $15 \%$. For case 3 , spot 1 is on the primary and spot 2 is on the secondary. For a relatively big spot 2 , we assume that the spot 2 latitude is $90^{\circ}$. Only to do so, it can produce the larger distortional effect required. The remaining spot 2 parameter subset, the spot 1 parameter subset, and the orbital parameter subset converge. For case4, we assume that the spot 1 and spot 2 latitudes are $90^{\circ}$ for the same reason. The 
Table 2. The results of LC analysis in 2004.

\begin{tabular}{|c|c|c|c|c|}
\hline Element & case1 & case2 & case3 & case4 \\
\hline$T_{1}$ & $6100 \mathrm{~K} a$ & $6100 \mathrm{~K} a$ & $6100 \mathrm{~K} a$ & $6100 \mathrm{~K} a$ \\
\hline$q$ & $0.735 a$ & $0.735 a$ & $0.735 a$ & $0.735 a$ \\
\hline$i$ & $85.57^{\circ} a$ & $85.57^{\circ} a$ & $85.57^{\circ} a$ & $85.57^{\circ} a$ \\
\hline$T_{2}$ & $4896 \pm 3 \mathrm{~K}$ & $4947 \pm 4 \mathrm{~K}$ & $4900 \pm 4 \mathrm{~K}$ & $4947 \pm 5 \mathrm{~K}$ \\
\hline$\Omega_{1}$ & $3.866 \pm 0.004$ & $3.881 \pm 0.004$ & $3.873 \pm 0.004$ & $3.872 \pm 0.004$ \\
\hline$\Omega_{2}$ & $4.467 \pm 0.004$ & $4.477 \pm 0.004$ & $4.468 \pm 0.004$ & $4.452 \pm 0.004$ \\
\hline$L_{1 B} /\left(L_{1}+L_{2}\right)_{B}$ & $0.9036 \pm 0.0006$ & $0.8964 \pm 0.0006$ & $0.9026 \pm 0.0006$ & $0.8956 \pm 0.0007$ \\
\hline$L_{1 V} /\left(L_{1}+L_{2}\right)_{V}$ & $0.8683 \pm 0.0009$ & $0.8609 \pm 0.0009$ & $0.8672 \pm 0.0009$ & $0.8598 \pm 0.0010$ \\
\hline$L_{1 I} /\left(L_{1}+L_{2}\right)_{I}$ & $0.8143 \pm 0.0012$ & $0.8077 \pm 0.0012$ & $0.8131 \pm 0.0012$ & $0.8063 \pm 0.0013$ \\
\hline$r_{1}($ pole $)$ & $0.3160 \pm 0.0004$ & $0.3144 \pm 0.0004$ & $0.3152 \pm 0.0004$ & $0.3153 \pm 0.0004$ \\
\hline$r_{1}$ (point) & $0.3457 \pm 0.0006$ & $0.3434 \pm 0.0006$ & $0.3446 \pm 0.0006$ & $0.3447 \pm 0.0006$ \\
\hline$r_{1}$ (side) & $0.3252 \pm 0.0004$ & $0.3235 \pm 0.0004$ & $0.3244 \pm 0.0005$ & $0.3245 \pm 0.0005$ \\
\hline$r_{1}$ (back) & $0.3367 \pm 0.0005$ & $0.3347 \pm 0.0005$ & $0.3358 \pm 0.0005$ & $0.3359 \pm 0.0005$ \\
\hline$r_{2}($ pole $)$ & $0.2189 \pm 0.0003$ & $0.2183 \pm 0.0003$ & $0.2189 \pm 0.0003$ & $0.2199 \pm 0.0002$ \\
\hline$r_{2}$ (point) & $0.2281 \pm 0.0003$ & $0.2274 \pm 0.0003$ & $0.2281 \pm 0.0003$ & $0.2293 \pm 0.0003$ \\
\hline$r_{2}$ (side) & $0.2217 \pm 0.0003$ & $0.2210 \pm 0.0003$ & $0.2216 \pm 0.0003$ & $0.2227 \pm 0.0003$ \\
\hline$r_{2}$ (back) & $0.2263 \pm 0.0003$ & $0.2255 \pm 0.0003$ & $0.2262 \pm 0.0003$ & $0.2274 \pm 0.0003$ \\
\hline Latitude $_{\text {spot } 1}$ & $23^{\circ} \pm 6^{\circ}$ & $88^{\circ} \pm 11^{\circ}$ & $21.6^{\circ} \pm 1.6^{\circ}$ & $90^{\circ} a$ \\
\hline Longitude $_{\text {spot } 1}$ & $86^{\circ} \pm 8^{\circ}$ & $85^{\circ} \pm 5^{\circ}$ & $83.5^{\circ} \pm 0.9^{\circ}$ & $84.3^{\circ} \pm 1.8^{\circ}$ \\
\hline Radius $_{\text {spot1 }}$ & $24^{\circ} \pm 1^{\circ}$ & $64^{\circ} \pm 6^{\circ}$ & $24.1^{\circ} \pm 1.3^{\circ}$ & $55.0^{\circ} \pm 1.0^{\circ}$ \\
\hline Temperature $_{\text {spot } 1}$ & $4181 \mathrm{~K} \pm 108 \mathrm{~K}$ & $4600 \mathrm{~K} \pm 20 \mathrm{~K}$ & $3631 \mathrm{~K} \pm 129 \mathrm{~K}$ & $4506 \mathrm{~K} \pm 21 \mathrm{~K}$ \\
\hline Latitude $_{\text {spot } 2}$ & $22^{\circ} \pm 1^{\circ}$ & $20.1^{\circ} \pm 0.5^{\circ}$ & $90^{\circ} a$ & $90^{\circ} a$ \\
\hline Longitude $_{\text {spot2 }}$ & $316^{\circ} \pm 4^{\circ}$ & $315.6^{\circ} \pm 0.3^{\circ}$ & $312.3^{\circ} \pm 0.6^{\circ}$ & $313.8^{\circ} \pm 0.5^{\circ}$ \\
\hline Radius $_{\text {spot2 }}$ & $26^{\circ} \pm 2^{\circ}$ & $27.1^{\circ} \pm 0.2^{\circ}$ & $59.8^{\circ} \pm 2.2^{\circ}$ & $61.9^{\circ} \pm 0.6^{\circ}$ \\
\hline Temperature $_{\text {spot } 2}$ & $3941 \mathrm{~K} \pm 79 \mathrm{~K}$ & $3701 \mathrm{~K} \pm 101 \mathrm{~K}$ & $4426 \mathrm{~K} \pm 23 \mathrm{~K}$ & $4484 \mathrm{~K} \pm 14 \mathrm{~K}$ \\
\hline$\Sigma \omega_{i}(\mathrm{O}-\mathrm{C})_{i}^{2}$ & 0.1249 & 0.1459 & 0.1464 & 0.1558 \\
\hline
\end{tabular}

Parameters not adjusted in the solution are denoted by a mark " $a$ ".
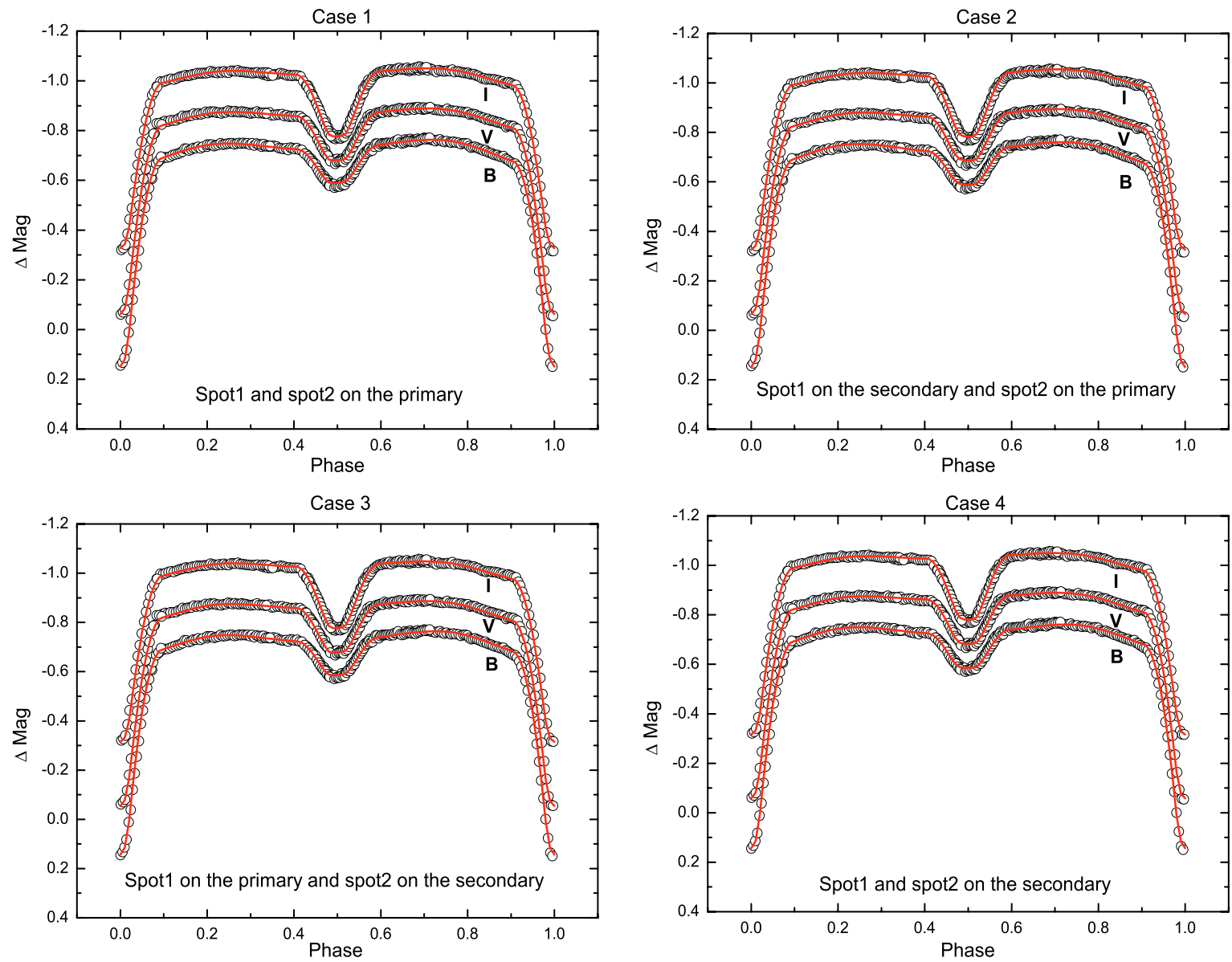

Fig. 2. The observational and theoretical light curves of RT And in 2004. The circles and solid lines represent the observational and theoretical light curves, respectively. 

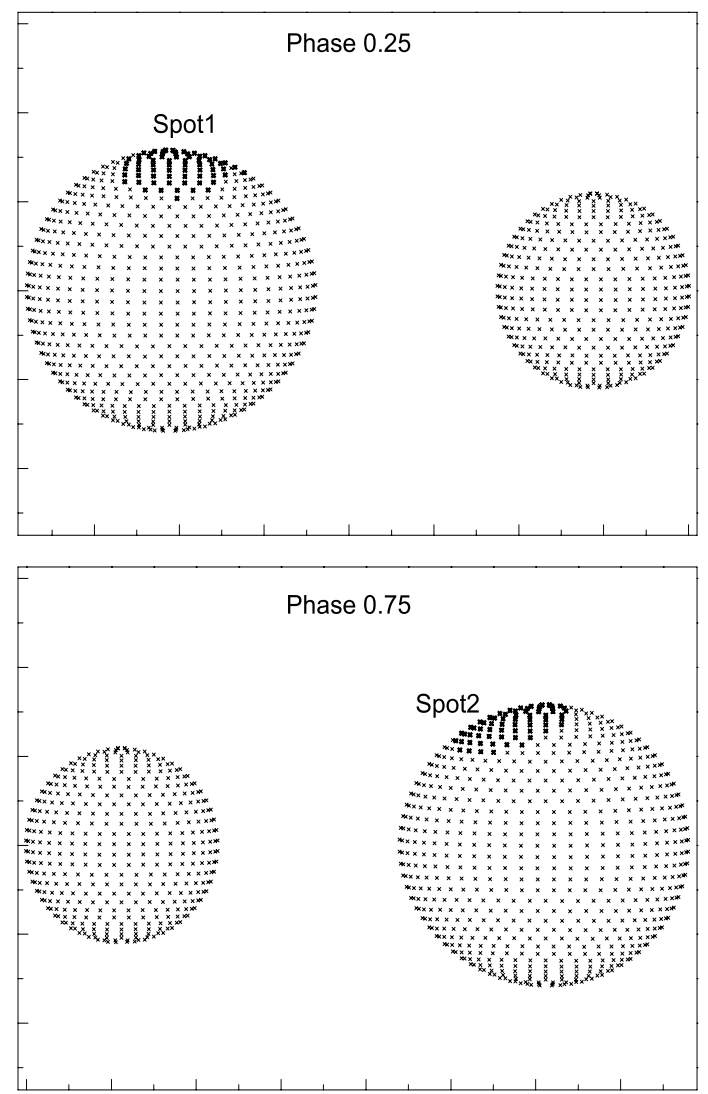

Fig. 3. The configurations of RT And in phases 0.25 and 0.75 in 2004.

remaining spot 1 parameter subset, the remaining spot 2 parameter subset, and the orbital parameter subset converge.

As can be seen from Fig. 2 where in case 3 and case 4 spot 2 is located on the secondary, the fitting is not good on the shoulders of the primary eclipse. For case 1 and case 2 where the spot 2 is located on the primary, the fitting is good not only in the eclipse but also outside the eclipse, while the weighted sum of squares of residuals for the LCs of case1 is smaller than that of case2, so we conclude case 1 of the two spots being on the primary star of RT And is most successful for describing the distortion of the LCs in 2004. Corresponding configurations of RT And for the best solution (case1) in phases 0.25 and 0.75 are shown in Fig. 3.

\subsection{Analysis of the light curve in 2005 and 1999}

As can be seen from Fig. 1, both of the LCs in 2005 and 1999 do not have sufficient phase coverage. But we still can derive the spot parameters from the best fit on the basis of the best photometric solution in 2004. We fix the orbital parameters and only adjust the spot parameters.

Comparing the LCs in 2005 with those in 2004 shows that the spot 2 becomes feeble or even disappears in Jan. 2005, and a new spot (spot3) appears around the secondary minimum or it might be spot1. (This seems to be impossible because its longitude changes quite large (about 0.3 phase).) From a comparison between the LCs in 2005 and the clean theoretical LCs, spot2 might exist and become slight.

Pribulla et al. (2000) find that the positions of the spots within the "belt" around $270^{\circ}$ seem to be periodic and that the period was $6.8 \pm 0.5$ years, which was obtained by the differential corrections method assuming a quadratic trend combined with a
Table 3. The analysis results in 2005 and 1999.

\begin{tabular}{lclc}
\hline \hline \multirow{2}{*}{2005} & & & \multicolumn{2}{c}{1999} \\
Element & Value & Element & Value \\
\hline Latitude $_{\text {spot3 }}$ & $33^{\circ} \pm 11^{\circ}$ & Latitude $_{\text {spot4 }}$ & $74^{\circ} \pm 28^{\circ}$ \\
Longitude $_{\text {spot3 }}$ & $181^{\circ} \pm 6^{\circ}$ & Longitude $_{\text {spot4 }}$ & $68^{\circ} \pm 6^{\circ}$ \\
Radius $_{\text {spot3 }}$ & $38^{\circ} \pm 2^{\circ}$ & Radius $_{\text {spot4 }}$ & $16^{\circ} \pm 8^{\circ}$ \\
Temperature $_{\text {spot3 }}$ & $5573 \mathrm{~K} \pm 122 \mathrm{~K}$ & Temperature $_{\text {spot4 }}$ & $3474 \mathrm{~K} \pm 159 \mathrm{~K}$ \\
Latitude $_{\text {spot2 }}$ & $16^{\circ} \pm 3^{\circ}$ & Latitude $_{\text {spot5 }}$ & $26.0^{\circ} \pm 0.4^{\circ}$ \\
Longitude $_{\text {spot2 }}$ & $316^{\circ} a$ & Longitude $_{\text {spot5 }}$ & $292^{\circ} a$ \\
Radius $_{\text {spot2 }}$ & $26^{\circ} a$ & Radius $_{\text {spot5 }}$ & $30.0^{\circ} \pm 0.2^{\circ}$ \\
Temperature $_{\text {spot2 }}$ & $3941 \mathrm{~K} a$ & Temperature $_{\text {spot5 }}$ & $3269 \mathrm{~K} \pm 231 \mathrm{~K}$ \\
\hline$\Sigma \omega_{i}(\mathrm{O}-\mathrm{C})_{i}^{2}$ & 0.0926 & & 0.218 \\
\hline
\end{tabular}

Parameters not adjusted in the solution are denoted by a mark " $a$ ".

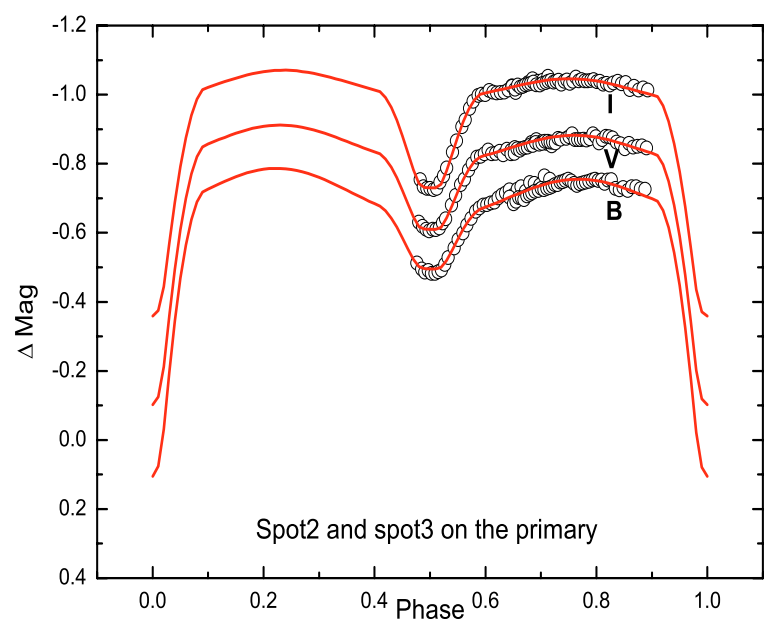

Fig. 4. The observational and theoretical light curves of RT And in 2005. The circles and solid lines represent the observational and theoretical light curves, respectively.

sine wave. If the longitude of the spot around $270^{\circ}$ confirms the rule, the spot 2 longitude changes are quite small on a time scale of two months, so we fix the longitude. From the short timescale observations (LC9 and LC10) by Macuso et al. (1979a) and from the October observations and the December observations (LC17 and LC18) by Dapergolas et al. (1991) and Gordon et al. (1990), respectively (see Pribulla et al. 2000) there was no similar rule for other parameters of the same spot (spot area, latitude, and temperature). For the other short-period RS CVn system XY UMa, Pribulla et al. (2001) identified the same spots on the subsequent LCs. From their results, we conclude that the area and temperature of the same spot change very little over a short time interval, one month or so, on condition that the spot latitude is fixed. Because a spot area is highly correlated with its temperature and the latitude is highly correlated with its radius, it is reasonable to only adjust one of the three spot parameters, so we assume the spot 2 area and temperature do not change and we only adjust the spot 2 latitude.

Spot 3 must be located on the primary component since the depression of the LCs of RT And occur around the secondary minimum where the secondary is eclipsed. Heckert et al. (1998) find that WY Can had secular variations during the secondary eclipse, but it was not explained by a spot model. For another short-period RS CVn system, XY UMa, Pribulla et al. (2001) find that the LCs 8 and 9 in 1999 showed equal maxima and the deep secondary minimum, and this behavior was explained by 

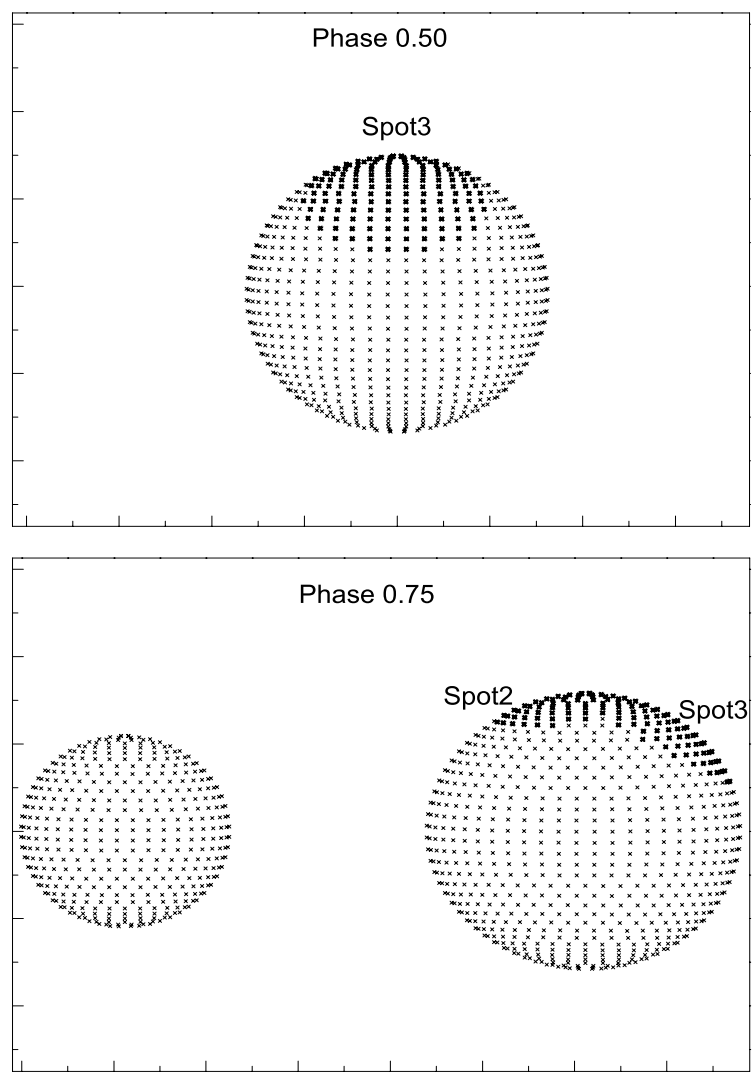

Fig. 5. The configurations of RT And in phases 0.50 and 0.75 in 2005.

putting a large cool starspot on the primary facing the observer at the secondary minimum.

We only adjust the spot 2 latitude and the spot 3 parameters. After some runs, the spot 2 latitude and the spot 3 parameters converge. The result is listed in Table 3, and the theoretical LCs for the spotted solution are shown in Fig. 4. Corresponding configurations of RT And in phases 0.50 and 0.75 are shown in Fig. 5.

We find two depressions from the 1999 LCs. The deepest depression in the phase range 0.05-0.3 (spot4) is approximately at phase 0.1 . The other deep depression in the phase range $0.8-0.95$ (spot5) is approximately at phase 0.81 , and the point is just the critical point of the observational data, so we fix the longitude of the spot5 as 0.81 . There is no information on the other parameters of the spots, so we only fix the spot5 longitude and adjust the other parameters. After some runs, we do not find the converged result because there are many free parameters and we only have the parts of LCs. In the course of our calculation, we find the spot parameters for the best fit (see Table 3). The theoretical LCs for the spotted solution are shown in Fig. 6. Corresponding configurations of RT And in phases 0.125 and 0.875 are shown in Fig. 7.

Even though the LCs in 1999 and 2005 are successfully explained using the spot model based on the photometric solution in 2004, the spot parameters might not be real because one small spot could affect more than half of the LC, and there are very large data gaps. However, all the spots do exist in 1999 and 2005 because their LC variations could be explained by the spot model, and these spots are only possibility for explaining these variations.

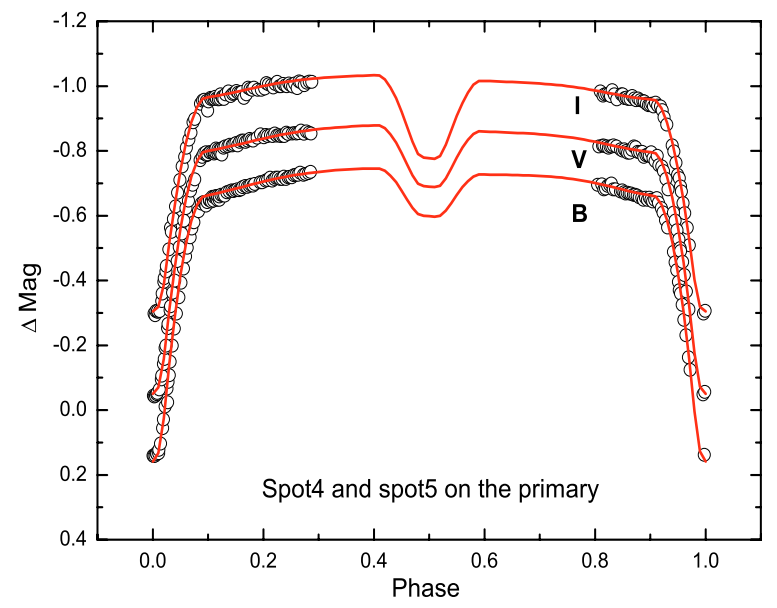

Fig. 6. The observational and theoretical light curves of RT And in 1999. The circles and solid lines represent the observational and theoretical light curves, respectively.
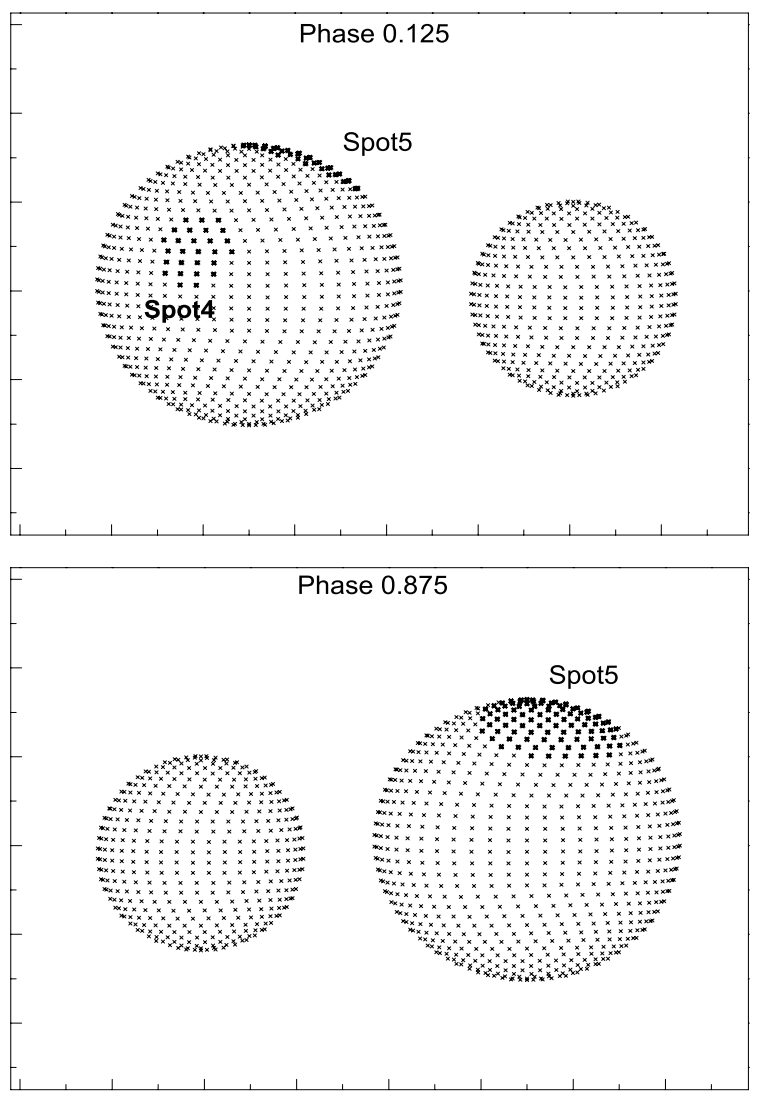

Fig. 7. The configurations of RT And in phases 0.125 and 0.875 in 1999.

\subsection{Analysis of the light curve variation}

Comparison of the LCs of 1999, 2004, and 2005 indicates short-term and long-term variations, especially the short-term variation. These variations are explained by the spot model. Comparing the LCs in 2005 with those in 2004 indicates fast dramatic LC variation on the time scale of two months during the secondary eclipse. The maximum variations are 0.095 , 0.075 and $0.050 \mathrm{mag}$ in $B, V$, and $I$ bands, respectively. Another significant LC change within one and a half months was registered only for $V$ band in October-December 1974 by 
Mancuso et al. (1979a). For their observations, the active longitude belt is near phase 0.75 ; however, for our observations, the dramatic active region is near phase 0.5 , so the most significant LC variation on the time scale of the two months is detected around the secondary minimum.

On Aug. 26-Sep. 14 1999, Pribula et al. (2000) obtained most parts of the LCs of RT And. The interval between their observation and ours in 1999 is only about one and half months. For our observation, Fig. 1 shows that the LCs of RT And during 1999 increase almost linearly after the primary eclipse. This behavior is different from the one derived by Pribulla et al. (2000) that the brightness does not change basically. This indicates a variability in the spot extent on a time scale of one and half months.

From the October and the December observations (LC17 and LC18) by Dapergolas et al. (1991) and Gordon et al. (1990), respectively, the magnitude of the LC disturbance changes quite rapidly (see Pribulla et al. 2000). This indicates a fast variability for the starspot on the time scale of two months.

The above does indicate fast variability for the starspot on a short-time scale interval, one and half months or so. This also suggests the lifetime of the starspot might be much shorter, one month or even several weeks.

\section{Discussion and conclusions}

New CCD $B, V, R$, and I LCs of RT And obtained from 1999 to 2005 are presented and included in a comprehensive study.

The four simultaneous solutions of BVI LCs in 2004 we are derived. From these results we find that the case of two spots being on the primary star is most successful in representing the distortion of the LCs. The contribution of the primary star of RT And to the total light is 0.90 in $B, 0.87$ in $V$, and 0.81 in the $I$ band. These values are the same as those of Budding \& Zeilik (1987), Zeilik et al. (1989), and Pribulla et al. (2000), while Wang \& Lu (1993) and Kjurkchiev et al. (2001) obtained slightly lower values. This might be caused by the high orbital inclination. Our observed secondary minima of RT And favors the high orbital inclination corresponding to total eclipsing.

Comparing the LCs of 1999, 2004, and 2005, it is clear that the relative magnitude of the LC distortion changes on short and long time scales. Although the LCs in 1999 and 2005 do not have sufficient phase coverage, on the basis of the photometric solution of 2004 we can still use the spot model to explain the change successfully.

From the configurations of RT And, it is evident that cool active regions are over the stellar pole. Of course, the spot latitudes determined by the traditional LC method are usually not unique, and thus less reliable. But the spot2 latitude might be reliable because the latitude of a spot could be reliably obtained for an eclipsed spot. It is well known that spot latitudes can be directly recovered from Doppler imaging. Out of 65 stars whose surfaces were mapped with the Doppler imaging technique, 36 stars showed prominent polar spots (Strassmeier 2002), so it is reasonable that cool active regions are over the stellar pole of RT And. High-resolution spectroscopic observations are needed to solve the controversy of polar spots.

A representative sample of the starspot temperatures for active dwarfs, giants, and subgiants were collected and plotted by Berdyugina (2005), who finds a clear tendency for spots to be more contrasting with respect to the photosphere in the hotter stars. The temperature difference between spots and the photosphere decreases from about $2000 \mathrm{~K}$ in G0 stars to $200 \mathrm{~K}$ in M4 stars, and there seemed to be no difference in this property

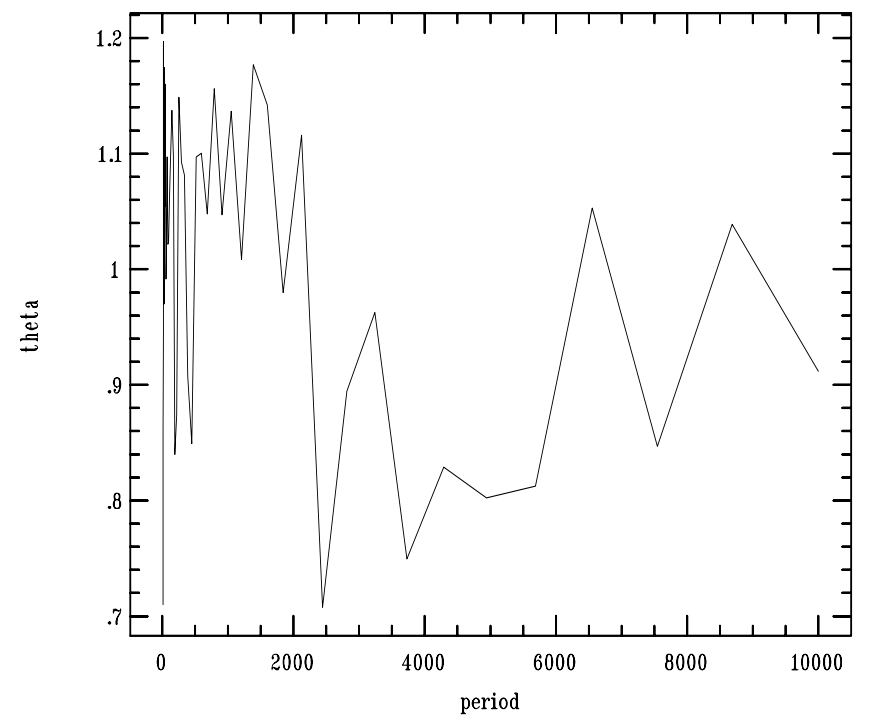

Fig. 8. Periodogram of the longitude of spots around $270^{\circ}$ where the vertical axis is the phase dispersion statistic (theta) and the unit of the horizontal axis (period) is one day. The minimum corresponds to the period $p=2442 \mathrm{~d}$.

between active dwarfs and giants, at least for G-K stars. This implies the nature of starspots is the same in all active stars. If all active stars follow the relation for the primary (F8-G0) of RT And, it might be accepted that the starspot temperature is about $2000 \mathrm{~K}$ below the photosphere, especially for the starspots for our best result in 2004.

Zeilik et al. (1989) found that active regions on the primary tend to appear in two active longitude belts, one near $90^{\circ}$, the other near $270^{\circ}$. New and more extensive parameters of the spots confirm this hypothesis. Six of 18 spot longitudes are in the range $45^{\circ}-135^{\circ}$, and 7 of 18 spot longitudes are in the range $225^{\circ}-315^{\circ}$ (see Pribulla et al. 2000). By now, 10 of 29 spot longitudes are in the range $45^{\circ}-135^{\circ}, 10$ of 29 spot longitudes are in the range $225^{\circ}-315^{\circ}$. Thus, for RT And, there is clear evidence of active longitudes near the quadratures. According to Jeffers (2005), heavily spotted stellar surface can possibly cause spurious active-longitude detections at the quadrature points of the eclipsing binaries. The presence of active longitudes near the quadrature points of RT And could indicate that the surface is peppered with many spots whose sizes are below the resolving capabilities of the present diagnostic techniques for studying starspots. Pribulla et al. (2000) hypothesize that the belts around $90^{\circ}$ and $270^{\circ}$ could join later and form one wide belt around longitude $180^{\circ}$. The belt was not found in our observations in 2004. For our observation in 2005, however, a large cool starspot around the longitude $180^{\circ}$ was.

We have collected the values of the longitude of spots within the belt around $270^{\circ}$ derived by Pribulla et al. (2000), besides the value $237^{\circ}$ at HJD 2451034 derived by Kjurkchieva et al. (2001) and $316^{\circ}$ at HJD 2453314 obtained by us. These values were checked for periodicity using the the phase-dispersion minimization method (Stellingerf 1978) in the range 10-10000 days. Figure 8 shows the phase dispersion statistic(theta) vs. period, and the phase dispersion minimization is at 2442 day (6.69 years). The best periodicity $P=6.69$ years coincides with the value obtained by using the differential correction method assuming quadratic trend combined with a sine wave (Pribulla et al. 2000). To estimate the error of the period, we do an additional fit using the sine algorithm, because these values 
possibly show a periodic oscillation around $270^{\circ}$. We find a $1 \sigma$ uncertainty of the period of about 0.8 years, so we adopt $P=6.69 \pm 0.80$.

Pribulla et al. (2003) think that RT And is a rather inactive system - the amplitude of the photometric wave is usually lower than 0.05 mag in spite of a high orbital inclination. For our observations, however, it is found that the most significant LCs variation occurs on the time scale of the two months around the secondary minimum. The maximum variation is $0.095,0.075$, and $0.050 \mathrm{mag}$ in the $B, V$, and $I$ bands, respectively. This behavior is explained by a large cool starspot facing the observer around the secondary minimum. This indicates a fast variation in the spot's extent on the time scale of months or even weeks. For the evaluation of the total distortion of the LCs, Pribulla et al. (2000) introduced a dimensionless factor $\beta$ defined as the ratio between the luminosity blocked by spots and the total luminosity of an unspotted binary. The resulting spot factor obtained for the LCs in 2004 is 0.0645 . The resulting spot factor of the LCs observed by Kjurkchieva et al. (2001) in 1998 is 0.0293. These spot factors are much larger than those obtained by Pribulla et al. (2000). They change quite rapidly, so RT And should be a moderately active system.

A large number of simultaneous photometric and spectroscopic observations for RT And are very desirable for understanding stellar activity better and determining which component the activity is from, the primary or the secondary. We shall make photometric and spectroscopic observations simultaneously for the short-period group of RS CVn systems on time scale of months even weeks. Then, we shall study the photospheric activity and chromospheric activity by analyzing the LCs and spectral lines, respectively, and also explore the spatial correlation between photospheric activity and chromospheric activity. We shall also study their evolution on different time scales and stellar activity cycles.

Acknowledgements. We would like to thank Dr. Xiao-bin Wang for helping us with the IRAF package, along with Mr. Xi-liang Zhang and the observational assistants of 1 meter telescope team of Yunnan Observatory for their help and support during our observations. we would also like to thank the anonymous referee for his (her) valuable suggestions and comments, which led to large improvement in the manuscript. This work is supported by the NSFC under grant No. 10373023.

\section{References}

Agerer, F., \& Hübscher, J. 1999, Inf. Bull. Var. Stars No. 4711 Arévalo, M. J., Lázaro, C., \& Claret, A. 1995, AJ, 110, 1376

Bakiş, V., Tüysüz, M., Zejda, M., et al. 2003, Inf. Bull. Var. Stars, 5399 Berdyugina, S. V. 2005, Living Rev. Sol. Phys., 2, 8 [Online Article] Bíró I, B., \& Borkovits, T. 2000, Inf. Bull. Var. Stars, 4967 Borkovits, T., \& Bíró, I. B. 1998, Inf. Bull. Var. Stars, 4633 Borkovits, T., Bíró, I. B., \& Kovács, T. 2001, Inf. Bull. Var. Stars, 5206 Borkovits, T., Bíró, I. B., Csizmadia, S., et al. 2004, Inf. Bull. Var. Stars, 5579 Budding, E., \& Zeilik, M. 1987, ApJ, 319, 827

Dapergolas, A., Kontizas, E., \& Kontizas, M. 1991 Inf. Bull. Var. Stars, 3661 Dean, C. A. 1974, PASP, 86, 912

Dumitrescu, A. 1974, Studii si Cercetari de Astr., 18, 47

Ekmekçi, F., Ak, H. 2001, Inf. Bull. Var. Stars, 5174

Gordon, K. 1948, AJ, 53, 198

Gordon, K. 1955, AJ, 60, 422

Gordon, S., Hall, S., Ledlow, M., et al. 1990, Inf. Bull. Var. Stars, 3469

Heckert, P. A. 1995, Inf. Bull. Var. Stars, 4224

Heckert, P. A. 1996, Inf. Bull. Var. Stars, 4384

Heckert, P. A. 1998, Inf. Bull. Var. Stars, 4656

Heckert, P. A., Maloney, G. V., Stewart, M. C., et al. 1998, AJ, 115, 1145

Jeffers, S. V. 2005, MNRAS, 359, 729

Johnson, H. L. 1966, ARA\&A, 4, 193

Kjurkchieva, D. P., Marchev, D. V., \& Ogloza, W. 2001, A\&A, 378, 102

Kurucz, R. L. 1993, in light curve modeling of eclipsing Binary Stars, ed. E. F. Milone (New York: Springer-Verlag), 93

Lang, K. R. 1992, Astrophysical data: Planets and Stars (Springer: New York), 138

Mancuso, S., Milano, L., Russo, G., et al. 1979a, A\&AS, 36, 415

Mancuso, s., Milano, L., Russo, G., et al. 1979b, Ap\&SS, 66, 475

Milano, L., Russo, G., \& Mancuso, S. 1981, A\&A, 103, 57

Nelson, R. H. 2000, Inf. Bull. Var. Stars, 4840

Nelson, R. H. 2003, Inf. Bull. Var. Stars, 5371

Payne-Gaposchkin, C. 1946, ApJ, 103, 291

Popper, D. M. 1994, AJ, 108, 1091

Pribulla, T., Chochol, D., \& Parimucha, Š. 1999, Inf. Bull. Var. Stars, 4751

Pribulla, T., Chochol, D., Milano, L., et al. 2000, A\&A, 362, 169

Pribulla, T., Chochol, D., Heckert, P. A., et al. 2001, A\&A, 371, 997

Pribulla, T., Chochol, D., \& Vittone, A. A. 2003, Chin. J. Astron. Astrophys. Suppl., 3, 361

Stellingwerf, R. F. 1978, ApJ, 224, 953

Strassmeier, k. G. 2002, AN, 323, 309

Wang, X., \& Lu, W. 1993, in New Frontiers in Binary Star Research, ed. J. C. Leung, \& I. S. Nha, ASP Conf. Ser., 38, 280

Wilson, R. E., \& Devinney, E. J. 1971, ApJ, 166, 605

Wilson, R. E., \& Van Hamme, W. 2004, Computing Binary Star Observables, privately circulated monograph

Yakut, K., Erkan, N., Ulaş, B., et al. 2003, Inf. Bull Var. Stars, 5360

Zeilik, M., Cox, D. A., De Blasi, C., et al. 1989, ApJ, 345, 991 


\section{Online Material}


Table 1. The minimum times of RT And.

\begin{tabular}{|c|c|c|c|}
\hline 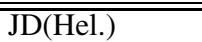 & $\overline{\overline{\text { Cycle }}}$ & $\overline{(\mathrm{O}-\mathrm{C})}$ & Source \\
\hline 2450682.4319 & -1297.5 & -0.0011 & Pribulla et al. (1999) \\
\hline 2450708.5334 & -1256.0 & 0.0002 & , \\
\hline 2450709.4772 & -1254.5 & 0.0003 & , \\
\hline 2450964.5073 & -849.0 & -0.0001 & Borkovits \& Bíró (1998) \\
\hline 2451015.4514 & -768.0 & 0.0008 & Ekmekçi \& Ak (2001) \\
\hline 2451032.4314 & -741.0 & -0.0003 & Kjurkchieva et al. (2001) \\
\hline 2451036.5189 & -734.5 & -0.0008 & , \\
\hline 2451041.5496 & -726.5 & -0.0016 & Pribulla et al. (1999) \\
\hline 2451066.3941 & -687.0 & 0.0003 & \\
\hline 2451076.4564 & -671.0 & -0.0003 & Agerer \& Hübscher (1999) \\
\hline 2451103.5007 & -628.0 & 0.0001 & . \\
\hline 2451142.4937 & -566.0 & -0.0005 & Pribulla et al. (1999) \\
\hline 2451150.3552 & -553.5 & -0.0006 & \\
\hline 2451417.3357 & -129.0 & -0.0002 & Pribulla et al. (2000) \\
\hline 2451426.4546 & -114.5 & -0.0008 & , \\
\hline 2451433.3732 & -103.5 & -0.0004 & , \\
\hline 2451434.3173 & -102.0 & 0.0003 & , \\
\hline 2451436.5185 & -98.5 & 0.0003 &, \\
\hline 2451437.4626 & -97.0 & 0.0010 & Ekmekçi \& Ak (2001). \\
\hline 2451438.4049 & -95.5 & -0.0001 & \\
\hline 2451463.2480 & -56.0 & 0.0003 & Bíró \& Borkovits (2000) \\
\hline 2451463.5634 & -55.5 & 0.0012 & \\
\hline 2451465.7640 & -52.0 & 0.0006 & Nelson (2000) \\
\hline 2451498.4680 & 0.0 & 0.0003 & Pribulla et al. (2000) \\
\hline 2451508.2166 & 15.5 & 0.0005 & , \\
\hline 2451511.3613 & 20.5 & 0.0006 & \\
\hline 2451530.2280 & 50.5 & -0.0006 & \\
\hline 2451792.4927 & 467.5 & 0.0010 & Yakut et al. (2003) \\
\hline 2451794.3793 & 470.5 & 0.0008 & , \\
\hline 2451815.4472 & 504.0 & -0.0004 & , \\
\hline 2451830.5434 & 528.0 & 0.0015 & , \\
\hline 2451863.2460 & 580.0 & -0.0002 & ” \\
\hline 2451884.3162 & 613.5 & 0.0009 & , \\
\hline 2451912.3028 & 658.0 & 0.0002 & , \\
\hline 2452120.4774 & 989.0 & -0.0005 & \\
\hline 2452186.5154 & 1094.0 & 0.0000 & Borkovits et al. (2001) \\
\hline 2452566.7024 & 1698.5 & -0.0003 & Nelson (2003) \\
\hline 2452860.4120 & 2165.5 & -0.0003 & Borkovits et al. (2004) \\
\hline 2453313.2401 & 2885.5 & -0.0007 & present paper \\
\hline 2453314.1839 & 2887.0 & -0.0003 & present paper \\
\hline 2453317.0140 & 2891.5 & -0.0003 & present paper \\
\hline 2451016.3973 & -766.5 & 0.0031 & Ekmekçi \& Ak (2001) \\
\hline 2451021.4303 & -758.5 & 0.0046 & Bíró \& Borkovits (2000) \\
\hline 2451160.4162 & -537.5 & -0.0027 & Pribulla et al. (1999) \\
\hline 2452577.3991 & 1715.5 & 0.0044 & Bakis et al. (2003) \\
\hline
\end{tabular}

